\title{
THE TRACE CLASS CONJECTURE AND THE WEYL UPPER BOUND ON THE DISCRETE SPECTRUM OF ARITHMETIC GROUPS
}

\section{LIZHEN Ji}

\section{Statement of results}

1.1. In this note, we announce two results: a solution to the trace class conjecture in the theory of Selberg trace formula and automorphic forms in complete generality (Theorem 1.3.3), and the sharp upper bound given by the Weyl law on the discrete spectrum of an arithmetic subgroup (Theorems 1.4.1 and 1.5.2). The latter result of the Weyl upper bound implies the trace class conjecture.

1.2. Let $\mathbf{G}$ be a semisimple algebraic group defined over $\mathbb{Q}$, and $\Gamma \subset \mathbf{G}(\mathbb{Q})$ an arithmetic subgroup. Then $G=\mathbf{G}(\mathbb{R})$ is a semisimple Lie group with finitely many connected components and with finite center. Let $K \subset G$ be a maximal compact subgroup, and $\mathfrak{g}=\mathfrak{k}+\mathfrak{p}$ be the Cartan decomposition of the Lie algebra $\mathfrak{g}$. The Killing form is positive definite on $\mathfrak{p}$ and negative definite on $\mathfrak{k}$. Define an inner product on $\mathfrak{g}$ that is equal to the Killing form on $\mathfrak{p}$ and the negative of the Killing form on $\mathfrak{k}$. Then this inner product defines a left $G$-invariant Riemannian metric on $G$. An important property of this metric is that it is invariant under the right $K$-multiplication.

Let $X=G / K$ be the symmetric space associated with $G$. By identifying the tangent space of $X$ at $K$ with $\mathfrak{p}$, the Killing form defines a $G$-invariant metric on $X=G / K$.

1.3. Denote the right regular representation of $G$ on $L^{2}(\Gamma \backslash G)$ by $R$. If $\Gamma \backslash G$ is compact, then $R$ is a direct sum of irreducible subrepresentations.

In the following, we assume that $\Gamma \backslash G$ is noncompact unless otherwise specified. Then $L^{2}(\Gamma \backslash G)$ admits the following orthogonal decomposition:

$$
L^{2}(\Gamma \backslash G)=L_{d}^{2}(\Gamma \backslash G) \oplus L_{c}^{2}(\Gamma \backslash G),
$$

where $L_{d}^{2}(\Gamma \backslash G)$ is the direct sum of irreducible subrepresentations of $R$ and $L_{c}^{2}(\Gamma \backslash G)$ is the orthogonal complement, which can be decomposed continuously according to Langlands' theory of Eisenstein series [17]. Denote the restriction of $R$ to the discrete subspace $L_{d}^{2}(\Gamma \backslash G)$ by $R_{d}$. A fundamental problem in the theory of automorphic forms is to understand the irreducible subrepresentations of $R_{d}$, and an important method for this problem is the Selberg trace formula.

Received January 17, 1997.

Partially Supported by a NSF postdoctoral fellowship 940727. 
For any $\alpha \in C_{0}^{\infty}(G)$, define an operator $R_{d}(\alpha)$ on $L_{d}^{2}(\Gamma \backslash G)$ by

$$
R_{d}(\alpha)=\int_{G} \alpha(g) R_{d}(g) d g
$$

The Selberg trace formula is an equality between the spectral and geometric expressions of the trace of $R_{d}(\alpha)$. An important longstanding problem in the theory of Selberg trace formula is the following conjecture (see $[2, \S 4.7][25, \S 4]$ $[29, \S 2.3][30$, Open problem 4] [12, p.14]).

Conjecture 1.3.1 (Trace Class Conjecture). For every $\alpha \in C_{0}^{\infty}(G)$, the operator $R_{d}(\alpha)$ on the discrete subspace $L_{d}^{2}(\Gamma \backslash G)$ is of the trace class.

Let $\mathcal{C}^{1}(G)$ be Harish-Chandra's Schwartz space of integrable rapidly decreasing functions (see [30, p.34] for definition). In [22, Corollary 0.2], Müller made a breakthrough and proved the following.

Theorem 1.3.2. If $\alpha \in \mathcal{C}^{1}(G)$ and is also $K$-finite, then $R_{d}(\alpha)$ is of the trace class.

Our first result is the following [15, Theorem 1.1.2].

Theorem 1.3.3. For every $\alpha \in \mathcal{C}^{1}(G), R_{d}(\alpha)$ is of the trace class; in particular, Conjecture 1.3.1 holds.

Müller gave a different proof of this theorem recently [21]. If the $\mathbb{Q}$ rank of $\mathbf{G}$ is equal to one, then Theorem 1.3.3 is due to Langlands [18].

1.4. Theorem 1.3.3 follows from polynomial upper bounds on the counting function of the discrete spectrum of $L_{d}^{2}(\Gamma \backslash G)$. Let $\Delta$ be the Beltrami-Laplace operator of $G$ with respect to the left $G$-invariant metric. Then $\Delta$ preserves the decomposition $L^{2}(\Gamma \backslash G)=L_{d}^{2}(\Gamma \backslash G) \oplus L_{c}^{2}(\Gamma \backslash G)$, and the restriction of $\Delta$ to $L_{d}^{2}(\Gamma \backslash G)$ has a discrete spectrum $\operatorname{Spec}_{d}(\Delta)=\left\{\lambda_{1}, \lambda_{2}, \cdots\right\}$ (repeated with multiplicity). For any $\lambda>0$, define the counting function of the discrete spectrum of $\Delta$ in $L_{d}^{2}(\Gamma \backslash G)$ by

$$
N_{d}(\lambda)=\left|\left\{\lambda_{i} \in \operatorname{Spec}_{d}(\Delta) \mid \lambda_{i} \leq \lambda\right\}\right| .
$$

In [2, Theorem 3], Borel \& Garland proved that $N_{d}(\lambda)$ is finite for every positive $\lambda$. Our second result is the following [15, Theorem 1.2.1].

Theorem 1.4.1. The counting function $N_{d}(\lambda)$ satisfies the Weyl upper bound, i.e.,

$$
\lim _{\lambda \rightarrow+\infty} \sup \frac{N_{d}(\lambda)}{\lambda^{n / 2}} \leq(4 \pi)^{-n / 2} \frac{\operatorname{vol}(\Gamma \backslash G)}{\Gamma\left(\frac{n}{2}+1\right)},
$$

where $n=\operatorname{dim} G$. 
Besides the application to the solution of the trace class conjecture above, Theorem 1.4.1 gives a quantitative description of the distribution of the irreducible subrepresentations of $R_{d}$ in $L_{d}^{2}(\Gamma \backslash G)$ and is an interesting result in and of itself.

If $\Gamma \backslash G$ is compact, then $\Delta$ has only a discrete spectrum, and the counting function $N_{d}(\lambda)$ satisfies the Weyl law, i.e., the equality holds in Theorem 1.4.1. On the other hand, if $\Gamma \backslash G$ is noncompact, it is conceivable that the Weyl law holds whenever $\Gamma$ is a congruence subgroup. But it is not known even for $\mathbf{G}=\mathrm{SL}(2)$. See the next section for discussions on related questions for locally symmetric spaces $\Gamma \backslash X$.

1.5. The study of the discrete spectrum of $\Gamma \backslash G$ is closely related to the study of the discrete spectrum of $\Gamma \backslash X$, and an important problem here concerns sharp bounds on the counting function of the discrete spectrum of the locally symmetric space $\Gamma \backslash X$.

For any irreducible unitary representation $\sigma$ of $K$, there is a homogeneous vector bundle $\tilde{E}_{\sigma}$ on $X$ and hence a locally homogeneous bundle $E_{\sigma}$ on $\Gamma \backslash X$. Denote the space of $L^{2}$-sections of the bundle $E_{\sigma}$ by $L^{2}(\Gamma \backslash X, \sigma)$. The Dirichlet quadratic form $D(f)=\int_{\Gamma \backslash X}|\nabla f|^{2}$ defines the Laplace operator $\Delta_{\sigma}$ on $L^{2}(\Gamma \backslash X, \sigma)$.

The space $L^{2}(\Gamma \backslash X, \sigma)$ also admits an orthogonal decomposition

$$
L^{2}(\Gamma \backslash X, \sigma)=L_{d}^{2}(\Gamma \backslash X, \sigma) \oplus L_{c}^{2}(\Gamma \backslash X, \sigma),
$$

where $\Delta_{\sigma}$ has a discrete spectrum $\operatorname{Spec}_{d}\left(\Delta_{\sigma}\right)$ on $L_{d}^{2}(\Gamma \backslash X, \sigma)$ and a continuous spectrum on $L_{c}^{2}(\Gamma \backslash X, \sigma)$. Denote the counting function of the discrete spectrum $\operatorname{Spec}_{d}\left(\Delta_{\sigma}\right)$ of $\Delta_{\sigma}$ by $N_{d, \sigma}(\lambda)$.

In $\left[2\right.$, Theorem 1], Borel \& Garland proved that $N_{d, \sigma}(\lambda)$ is finite for every positive $\lambda$. Suggested by the Weyl upper bound on the spectral counting function of the cuspidal subspace $L_{\text {cus }}^{2}(\Gamma \backslash X, \sigma)$ of $L_{d}^{2}(\Gamma \backslash X, \sigma)$ of Donnelly [5, Theorem $1.1]$, Borel \& Garland $[2, \S 4.7]$ raised the following.

Question 1.5.1. Decide whether $N_{d, \sigma}(\lambda)$ satisfies the Weyl upper bound,

$$
\lim _{\lambda \rightarrow+\infty} \sup \frac{N_{d, \sigma}(\lambda)}{\lambda^{d / 2}} \leq(4 \pi)^{-d / 2} \frac{\operatorname{vol}(\Gamma \backslash X)}{\Gamma\left(\frac{d}{2}+1\right)} \operatorname{dim} \sigma,
$$

where $d=\operatorname{dim} X$ and $\operatorname{dim} \sigma$ is the dimension of the representation space of $\sigma$.

Our second result is an affirmative answer to this question [16, Theorem 1.1.2].

Theorem 1.5.2. For any irreducible unitary representation $\sigma$ of $K$, the counting function $N_{d, \sigma}(\lambda)$ satisfies the Weyl upper bound:

$$
\lim _{\lambda \rightarrow+\infty} \sup \frac{N_{d, \sigma}(\lambda)}{\lambda^{d / 2}} \leq(4 \pi)^{-d / 2} \frac{\operatorname{vol}(\Gamma \backslash X)}{\Gamma\left(\frac{d}{2}+1\right)} \operatorname{dim} \sigma, \quad d=\operatorname{dim} X .
$$


Remark 1.5.3. We will use the proof of this theorem, but not this Weyl upper bound, to prove Theorem 1.4.1. In other words, the bound in Theorem 1.5.2 does not imply the bound in Theorem 1.4.1 and vice versa.

For $G=\operatorname{SL}(2, \mathbb{R})$, Selberg [28, p.668] proved the Weyl upper bound for $\Gamma \backslash X$ using the Selberg trace formula; Lax and Phillips [19, p. 205], and Colin de Verdiére [3] gave another proof using the pseudo-Laplace operator. For the general real rank one $G$, this upper bound in Theorem 1.5.2 is due to Donnelly [4, Theorem 1.1]. If $G=\operatorname{SL}(n, \mathbb{R})$ and $\Gamma \subset \operatorname{SL}(n, \mathbb{Z})$ is a congruence subgroup, $n \geq 3$, then the above bound follows from a result of Moeglin and Waldspurger $[20]$ on the description of the residual discrete spectrum and the bound on the cuspidal discrete spectrum in [5, Theorem 1.1].

There are some other partial results as well. If the $\Gamma$ rank of $\Gamma \backslash X$ is equal to one, which includes the $\mathbb{Q}$ rank one case, Donnelly [6, Theorem 4.11] and Langlands [18] independently proved that $N_{d, \sigma}(\lambda)$ has a ploynomial upper bound. For general $\Gamma \backslash X$, Müller [22, Theorem 0.1] proved that $N_{d, \sigma}(\lambda) \leq c\left(1+\lambda^{2 d}\right)$ for some positive constant $c$, which implies Theorem 1.3.2 above.

Based on this bound and the work of Selberg [28, p.668], Müller made the following conjecture in [23, Remark 2 on p.180].

Conjecture 1.5.4. Let $\Gamma$ be an arithmetic subgroup as above. Then $N_{d, \sigma}(\lambda)$ satisfies the Weyl law:

$$
\lim _{\lambda \rightarrow+\infty} \frac{N_{d, \sigma}(\lambda)}{\lambda^{d / 2}}=(4 \pi)^{-d / 2} \frac{\operatorname{vol}(\Gamma \backslash X)}{\Gamma\left(\frac{d}{2}+1\right)} \operatorname{dim} \sigma .
$$

In [27, Conjecture 2], Sarnak made a stronger conjecture that the counting function for the cuspidal discrete spectrum alone satisfies the Weyl law above. In view of the work of Efrat in [10] for arithmetic subgroups over function fields, it is very likely that the subgroup $\Gamma$ should be assumed to be a congruence subgroup in the above conjecture.

Conjecture 1.5.4 seems to be very difficult and is only known in the following cases:

(1) $\mathbf{G}=\mathrm{SL}(2), \Gamma$ a congruence subgroup (Selberg [28, p.668], Hejhal [13], and Huxley [14]).

(2) $\mathbf{G}=\mathrm{SO}(n, 1), X$ the real hyperbolic space, $\Gamma$ a congruence subgroup (Reznikov [24]).

(3) $\mathbf{G}=R_{k / \mathbb{Q}} \mathrm{SL}(2), \Gamma$ a congruence subgroup of the Hilbert modular group, where $R_{k / \mathbb{Q}}$ is the functor of the restriction of the ground field and $k$ is a totally real number field (Efrat [8] [9]).

In all the cases above, the Weyl law is proved in two steps:

(1) Use the Selberg trace formula to get the Weyl-Selberg law:

$$
N_{d, \sigma}(\lambda)+N_{c, \sigma}(\lambda) \sim(4 \pi)^{-d / 2} \frac{\operatorname{vol}(\Gamma \backslash X)}{\Gamma\left(\frac{d}{2}+1\right)} \operatorname{dim} \sigma \lambda^{d / 2},
$$


where $N_{c, \sigma}(\lambda)$ is the counting function of the continuous spectrum and is given by an integral of the determinant of the scattering matrices.

(2) Study the scattering matrices and show that the term $N_{c, \sigma}(\lambda)$ from the continuous spectrum is of smaller order than $\lambda^{d / 2}$ as $\lambda \rightarrow+\infty$.

In our proof of Theorem 1.5.2, we obtain an analogue of the Weyl-Selberg law (Theorems 2.3.2 and 2.4.1).

\section{Outline of the proofs}

2.1. Theorem 1.3.3 follows from Theorem 1.4 .1 by a now standard argument. The difficulty in proving Theorems 1.4.1 and 1.5.2 is the presence of a continuous spectrum of the Laplace operator and the fact that many eigenvalues are embedded in the continuous spectrum.

The basic idea of the proofs is to remove the continuous spectrum of the Laplace operator to get a pseudo-Laplace operator, which has only a discrete spectrum. We show that the counting function of the spectrum of the pseudoLaplace operator satisfies the Weyl law.

Then the problem is to understand the relation between the discrete spectrum of the Laplace operator and the spectrum of the pseudo-Laplace operator. It is in this step that we make essential use of Langlands' theory of Eisenstein series in [17].

2.2. Theorem 1.3.3 follows from Theorem 1.4 .1 in two steps (see $[15, \S 7]$ ):

(1) If $\alpha \in C_{0}^{2 n}(G)$ or $\alpha \in \mathcal{C}^{1}(G)$, then $R_{d}(\alpha)$ is a Hilbert-Schmidt operator.

(2) For any $\alpha \in \mathcal{C}^{1}(G)$, there exist $\beta \in \mathcal{C}^{1}(G)$ and $\mu, \nu \in C_{0}^{2 n}(G)$ such that $\alpha=\beta * \mu+\alpha * \nu$, and hence

$$
R_{d}(\alpha)=R_{d}(\beta) R_{d}(\mu)+R_{d}(\alpha) R_{d}(\nu) .
$$

Step 1 follows from the definition of the Hilbert-Schmidt norm and Theorem 1.4.1, and Step 2 is given in [30, Lemma 4.5] and follows from the existence of a good parametrix of powers of $\Delta$.

Theorem 1.4.1 depends on the proof of Theorem 1.5.2. We sketch the proofs of Theorem 1.5.2 in $\S 2.3$ and Theorem 1.4.1 in $\S 2.4$.

2.3. As mentioned earlier, the proof of Theorem 1.5.2 goes in two steps: (1) Show that the pseudo-Laplacian $\Delta_{T, \sigma}$ of $L^{2}(\Gamma \backslash X, \sigma)$ satisfies the Weyl law. (2) Bound the eigenvalues of the Laplace operator $\Delta_{\sigma}$ in terms of the eigenvalues of the pseudo-Laplace operator $\Delta_{T, \sigma}$.

First we define the pseudo-Laplace operator $\Delta_{T, \sigma}$. Let $H^{1}(\Gamma \backslash X, \sigma)$ be the space of sections $f$ of $E_{\sigma}$ over $\Gamma \backslash X$ such that $\int_{\Gamma \backslash X}|f|^{2}+|\nabla f|^{2}<+\infty$. Let $T$ be a large enough truncation parameter. Define $H_{T}^{1}(\Gamma \backslash X, \sigma) \subset H^{1}(\Gamma \backslash X, \sigma)$ to be the subspace of functions whose constant terms vanish above the height $T$ along every proper rational parabolic subgroup of $\mathbf{G}$. Then the Dirichlet quadratic form $D(f)=\int_{\Gamma \backslash X}|\nabla f|^{2}$ defines a self-adjoint operator on the closure of $H_{T}^{1}(\Gamma \backslash X, \sigma)$ in $L^{2}(\Gamma \backslash X, \sigma)$. This self-adjoint operator is the pseudo-Laplace 
operator associated with the height $T$ and denoted by $\Delta_{T, \sigma}$. Intuitively speaking, $\Delta_{T, \sigma}$ is obtained from $\Delta_{\sigma}$ by truncating off the constant terms above the height $T$. (see $[16, \S 3]$ for a precise definition of the pseudo-Laplace operator).

The pseudo-Laplace operator was first introduced by Lax and Phillips [19, p.206] and Colin de Verdiére [3] for $\Gamma \backslash X=\Gamma \backslash \mathrm{SL}(2, \mathbb{R}) / \mathrm{SO}(2, \mathbb{R})$. It was generalized to other spaces $\Gamma \backslash X$ by Müller [22]. An important property of the pseudo-Laplacian operator $\Delta_{T, \sigma}$ is that it has only a discrete spectrum.

Remark 2.3.1. The fact that the pseudo-Laplace operator has only a discrete spectrum can be explained as follows. In [11], Gelfand and Piatetski-Shapiro show that the Laplace operator has only a discrete spectrum on the cuspidal subspace $L_{c u s}^{2}(\Gamma \backslash X, \sigma)$ of $L^{2}(\Gamma \backslash X, \sigma)$, which is defined as the subspace of functions whose constant terms along every proper rational parabolic subgroup vanish identically. This means that the presence of the continuous spectrum comes from the constant terms. On the other hand, the decomposition principle in $[7$, Proposition 2.1] says that the continuous spectrum as a set does not change under compact perturbations. This implies that on the subspace of functions whose constant terms vanish in a neighborhood of infinity, the spectrum is discrete.

Using the precise reduction theory and the heat kernel method, we prove the following Weyl law for $\Delta_{T, \sigma}[16]$.

Theorem 2.3.2. Let $N_{T, \sigma}(\lambda)$ be the counting function of the spectrum of $\Delta_{T, \sigma}$. Then

$$
\lim _{\lambda \rightarrow+\infty} \frac{N_{T, \sigma}(\lambda)}{\lambda^{d / 2}}=(4 \pi)^{-d / 2} \frac{\operatorname{vol}(\Gamma \backslash X)}{\Gamma\left(\frac{d}{2}+1\right)} \operatorname{dim} \sigma,
$$

where $d=\operatorname{dim} \Gamma \backslash X$.

To understand the relation between the spectra of $\Delta_{\sigma}$ and $\Delta_{T, \sigma}$, we need to decompose $L^{2}(\Gamma \backslash X, \sigma)$ and $L_{d}^{2}(\Gamma \backslash X, \sigma)$ according to association classes of rational parabolic subgroups of $\mathbf{G}$.

Two rational parabolic subgroups of $\mathbf{G}$ are defined to be associated if their split components in the Langlands decomposition are conjugate under $G$. For each association class $\mathcal{C}$ of rational parabolic subgroups, there is a subspace $L_{\mathcal{C}}^{2}(\Gamma \backslash X, \sigma)$ of $L^{2}(\Gamma \backslash X, \sigma)$. Briefly speaking, this subspace is spanned by functions having only nonzero constant terms along parabolic subgroups in $\mathcal{C}$.

When $\mathcal{C}=\{\mathbf{G}\}, L_{\mathcal{C}}^{2}(\Gamma \backslash X, \sigma)$ is the cuspidal subspace $L_{\text {cus }}^{2}(\Gamma \backslash X, \sigma)$, i.e., the subspace spanned by functions whose constant terms vanish along every proper rational parabolic subgroup. The cuspidal subspace $L_{c u s}^{2}(\Gamma \backslash X, \sigma)$ is contained in $L_{d}^{2}(\Gamma \backslash X, \sigma)$ (see Remark 2.3.1 above), and its orthogonal complement in $L_{d}^{2}(\Gamma \backslash X, \sigma)$ is called the residual discrete subspace and denoted by $L_{r e s}^{2}(\Gamma \backslash X, \sigma)$, which is spanned by residues of Eisenstein series.

Every cuspidal eigenfunction of $\Delta_{\sigma}$ in $L_{c u s}^{2}(\Gamma \backslash X, \sigma)$ is clearly also an eigenfunction of the pseudo-Laplacian $\Delta_{T, \sigma}$, because truncating the constant terms has no effect on cuspidal functions. The problem is to understand the relation 
between the residual discrete spectrum of $\Delta_{\sigma}$ and the corresponding part of the spectrum of $\Delta_{T, \sigma}$.

The residual subspace $L_{\text {res }}^{2}(\Gamma \backslash X, \sigma)$ can be decomposed as follows:

$$
L_{\text {res }}^{2}(\Gamma \backslash X, \sigma)=\sum_{\mathcal{C} \neq\{\mathbf{G}\}} L_{d}^{2}(\Gamma \backslash X, \sigma) \cap L_{\mathcal{C}}^{2}(\Gamma \backslash X, \sigma),
$$

where $\mathcal{C}$ is over association classes of proper parabolic subgroups. Define $L_{d, \mathcal{C}}^{2}(\Gamma \backslash X, \sigma)=L_{\mathcal{C}}^{2}(\Gamma \backslash X, \sigma) \cap L_{d}^{2}(\Gamma \backslash X, \sigma)$. Our basic results concerning the relation between the spectra of $\Delta_{\sigma}$ and $\Delta_{T, \sigma}$ are as follows:

(1) When $\mathcal{C}$ is of rank one, i.e., the subgroups in $\mathcal{C}$ are of rank one, the eigenvalues of $\Delta_{\sigma}$ in $L_{d, \mathcal{C}}^{2}(\Gamma \backslash X, \sigma)$ can be uniformly approximated by the eigenvalues of $\Delta_{T, \sigma}$ on the corresponding subspace except for a subset of smaller order.

(2) When $\mathcal{C}$ is of rank greater than one, the discrete spectrum in $L_{d, \mathcal{C}}^{2}(\Gamma \backslash X, \sigma)$ is of smaller order than $\lambda^{d / 2}$, i.e., the Weyl law of $\Gamma \backslash X$.

More precisely, for each association class $\mathcal{C}$ of rank one, let $\mathbf{P}_{1}, \cdots, \mathbf{P}_{m}$ be representatives of $\Gamma$-conjugacy classes in $\mathcal{C}$. For each $\mathbf{P}_{i}$, let $P_{i}=N_{i} A_{i} M_{i}$ be the Langlands decomposition of $P_{i}$. Define $K_{i}=K \cap M_{i}$. Then $K_{i}$ is a maximal compact subgroup of $M_{i}$. The restriction of the representation $\sigma$ to $K_{i}$ is denoted by $\sigma_{i}$. Denote the spectral counting function of $L_{c u s}^{2}\left(\Gamma_{i} \backslash M_{i} / K_{i}, \sigma_{i}\right)$ by $N_{\text {cus }, i, \sigma_{i}}(\lambda)$. Denote the counting function of the (discrete) spectrum in $L_{d, \mathcal{C}}^{2}(\Gamma \backslash X, \sigma)$ by $N_{d, \mathcal{C}, \sigma}(\lambda)$. The decomposition of $L^{2}(\Gamma \backslash X, \sigma)$ according to the association classes also induces a decomposition of the pseudo-Laplace operator $\Delta_{T, \sigma}$. Denote the counting function of $\Delta_{T, \sigma}$ on the subspace associated with $\mathcal{C}$ by $N_{T, \mathcal{C}, \sigma}(\lambda)$. Then we have the following bounds from [16].

Proposition 2.3.3. Assume $\mathcal{C}$ is an association class of rank 1. Let $|\rho|$ be the norm of the half sum of positive roots of the parabolic subgroups in $\mathcal{C}$. Then

$$
N_{d, \mathcal{C}, \sigma}(\lambda) \leq N_{T, \mathcal{C}, \sigma}\left(\lambda+|\rho|^{2}\right)+\sum_{i=1}^{m} N_{c u s, i, \sigma_{i}}(\lambda) .
$$

By Donnelly's result in [5, Theorem 1.1] (or Theorem 1.5.2, since every cuspidal eigenfunction of $\Delta_{\sigma}$ is also an eigenfunction of $\Delta_{T, \sigma}$ ), the second term is of smaller order than $\lambda^{d / 2}$ since $\operatorname{dim} \Gamma_{i} \backslash M_{i} / K_{i}<\operatorname{dim} \Gamma \backslash X$.

Proposition 2.3.3 is proved as follows: For every rank one cuspidal Eisenstein series, using the positivity of the residue of rank one scattering matrices, we can construct an eigenfunction of the pseudo-Laplace operator $\Delta_{T, \sigma}$ for all the poles of the Eisenstein series in the right half plane except for at most one; and this eigenvalue of $\Delta_{T, \sigma}$ lies in a $|\rho|^{2}$-neighborhood of the corresponding residual eigenvalue of $\Delta_{\sigma}$ (see $\left.[16, \S 5]\right)$. 
Proposition 2.3.4. If $\mathcal{C}$ is of rank greater than 1 , then as $\lambda \rightarrow+\infty$,

$$
N_{d, \mathcal{C}, \sigma}(\lambda)=o\left(\lambda^{d / 2}\right) .
$$

Proposition 2.3.4 basically follows from Proposition 2.3.3 (or Theorem 2.3.2 to be more precise), the fact that the determinants of the scattering matrices are meromorphic functions of finite order [22, Theorem 5.10], and the factorization of higher rank scattering matrices into rank one scattering matrices of some groups of smaller dimension than $G$ (see $[16, \S 2.7]$ ). This factorization plays an important role in the meromorphic continuation of Eisenstein series in [17] [12].

Together with Theorem 2.3.2 and the fact observed above that every cuspidal eigenfunction of $\Delta_{\sigma}$ is also an eigenfunction of $\Delta_{T, \sigma}$, Propositions 2.3.3 and 2.3.4 prove Theorem 1.5.2.

2.4. Theorem 1.4.1 is proved similarly as Theorem 1.5.2. But we need some auxiliary spaces in order to bound the error terms which arise in approximating the discrete spectrum of the Laplace operator by the spectrum of the pseudoLaplace operator.

We can also define the pseudo-Laplace operator $\Delta_{T}$ for the Laplace operator $\Delta$ on $L^{2}(\Gamma \backslash G)$ and prove that $\Delta_{T}$ satisfies the Weyl law [15].

Theorem 2.4.1. Let $N_{T}(\lambda)$ be the counting function of the spectrum of the pseudo-Laplacian $\Delta_{T}$ of $\Gamma \backslash G$. Then

$$
\lim _{\lambda \rightarrow+\infty} \frac{N_{T}(\lambda)}{\lambda^{n / 2}}=(4 \pi)^{-n / 2} \frac{\operatorname{vol}(\Gamma \backslash G)}{\Gamma\left(\frac{n}{2}+1\right)},
$$

where $n=\operatorname{dim} \Gamma \backslash G$.

As in the proof of Theorem 1.5.2, the problem is to relate the residual discrete spectrum of the Laplacian $\Delta$ to the corresponding part of the spectrum of the pseudo-Laplacian $\Delta_{T}$. For this purpose, we use the right $K$-action on $\Gamma \backslash G$ to reduce it to the case of $\Gamma \backslash X$ above. Since the Riemannian metric of $\Gamma \backslash G$ is right $K$-invariant, we can decompose both $\Delta$ and $\Delta_{T}$ according to this action of $K$. More precisely,

$$
L^{2}(\Gamma \backslash G)=\sum_{\sigma \in \hat{K}}(\operatorname{dim} \sigma) L^{2}(\Gamma \backslash X, \sigma),
$$

where $\hat{K}$ is the set of irreducible unitary representations of $K$, and for any association class $\mathcal{C}$ of proper rational parabolic subgroups,

$$
L_{\mathcal{C}}^{2}(\Gamma \backslash G)=\sum_{\sigma \in \hat{K}}(\operatorname{dim} \sigma) L_{\mathcal{C}}^{2}(\Gamma \backslash X, \sigma),
$$

and hence

$$
N_{d, \mathcal{C}}(\lambda)=\sum_{\sigma \in \hat{K}}(\operatorname{dim} \sigma) N_{d, \mathcal{C}, \sigma}(\lambda)
$$


where $N_{d, \mathcal{C}}(\lambda)$ is the counting function of the discrete spectrum in $L_{\mathcal{C}}^{2}(\Gamma \backslash G)$. If $\mathcal{C}$ is of rank one, then Proposition 2.3.3 shows that

$$
\begin{aligned}
N_{d, \mathcal{C}}(\lambda) & \leq \sum_{\sigma \in \hat{K}}(\operatorname{dim} \sigma)\left(N_{T, \mathcal{C}, \sigma}\left(\lambda+|\rho|^{2}\right)+\sum_{i=1}^{m} N_{c u s, i, \sigma_{i}}(\lambda)\right) \\
& =N_{T, \mathcal{C}}\left(\lambda+|\rho|^{2}\right)+\sum_{\sigma \in \hat{K}} \operatorname{dim} \sigma \sum_{i=1}^{m} N_{c u s, i, \sigma_{i}}(\lambda) .
\end{aligned}
$$

Then the problem is to show that the second term is of smaller order than $\lambda^{n / 2}$. Since $\sigma_{i}$ is the restriction of the representation $\sigma$ of $K$ to the proper subgroup $K_{i}, \sigma_{i}$ is not irreducible in general, and hence this second term in Equation (2.4.2) can not be bounded by the counting function of the cuspidal discrete spectrum of $L^{2}\left(\Gamma_{i} \backslash M_{i}\right)$ :

$$
N_{c u s, i}(\lambda)=\sum_{\delta \in \hat{K}_{i}}(\operatorname{dim} \delta) N_{c u s, i, \delta}(\lambda) .
$$

To overcome this problem, we introduce $K$-principal bundles $\Gamma_{i} \backslash B_{i}$ over the boundary spaces $\Gamma_{i} \backslash M_{i} / K_{i}$ and prove that the pseudo-Laplace operator of $\Gamma_{i} \backslash B_{i}$ also satisfies the Weyl law. In particular, the counting function of the cuspidal discrete spectrum of $L^{2}\left(\Gamma_{i} \backslash B_{i}\right)$ satisfies the upper bound given by the Weyl law. Since

$$
L_{c u s}^{2}\left(\Gamma_{i} \backslash B_{i}\right)=\sum_{\sigma \in \hat{K}}(\operatorname{dim} \sigma) L_{c u s}^{2}\left(\Gamma_{i} \backslash M_{i} / K_{i}, \sigma_{i}\right)
$$

and $\operatorname{dim} \Gamma_{i} \backslash B_{i}<\operatorname{dim} \Gamma \backslash G$, we can show that the second term in Equation (2.4.2) is of smaller order than $\lambda^{n / 2}$.

We also need these $K$-principal bundles $\Gamma_{i} \backslash B_{i}$ to establish the analogous bound in Proposition 2.3.4 that the counting function of the discrete spectrum of $L_{\mathcal{C}}^{2}(\Gamma \backslash G)$ is of smaller order than $\lambda^{n / 2}$ when $\mathcal{C}$ is of rank greater than 1 . For details, see [15].

\section{Acknowledgements}

I would like to thank Professor S. T. Yau for suggesting me to study [22] and Professor W. Müller for his interests in early partial results, which encouraged me to complete the work in $[16]$. 


\section{References}

1. J. Arthur, The trace formula and Hecke operators, in Number theory, trace formulas and discrete groups, ed. by K. Aubert et al., Academic Press, 1989, pp. 11-27.

2. A. Borel and H. Garland, Laplacian and the discrete spectrum of an arithmetic group, Amer. J. Math. 105 (1983), 309-335.

3. Y. Colin de Verdiére, Pseudo-Laplaciens II, Ann. Inst. Fourier 33 (1983), 87-113.

4. H. Donnelly, On the point spectrum for finite volume symmetric spaces of negative curvature, Comm. Partial Diff. Eq. 6 (1981), 963-992.

5. _ On the cuspidal spectrum for finite volume symmetric spaces, J. Diff. Geom. 17 (1982), 239-253.

6. _ Eigenvalue estimates for certain noncompact manifolds, Michigan Math. J. 31 (1984), 349-357.

7. H. Donnelly and P. Li, Pure point spectrum and negative curvature for noncompact manifolds, Duke Math. J. 46 (1979), 497-503.

8. I. Efrat, The Selberg trace formula for $P S L_{2}(\mathbb{R})^{n}$, Mem. Amer. Math. Soc. 65 (1987), no. 359.

9. _ Cusp forms and higher rank, unpublished manuscript.

10. (1991), 125-129.

11. I. M. Gelfand and I. I. Piatetski-Shapiro, Unitary representations in homogeneous spaces with discrete stationary groups, Sov. Math. Dokl. 3 (1962), 1528-1531.

12. Harish-Chandra, Automorphic forms on semisimple Lie groups, Lect. Notes in Math., 62, Springer-Verlag, 1968.

13. D. Hejhal, The Selberg trace formula for $\operatorname{PSL}(2, R)$, vol. 2, Lecture Notes in Math., 1001, Springer-Verlag, 1983.

14. M. Huxley, Scattering matrices for congruence subgroups, in Modular forms, ed. by R. A. Rankin, Horwood, Chichester, 1984, pp. 141-156.

15. L. Ji, The trace class conjecture for arithmetic groups, preprint, 1996.

16. The Weyl upper bound on the discrete spectrum of locally symmetric spaces, J. Diff. Geom., (to appear).

17. R. Langlands, On the functional equations satisfied by Eisenstein series, Lecture Notes in Math. vol. 544, Springer-Verlag, 1976.

18. __ Rank-one residues of Eisenstein series, in Festschrift in honor of I. I. PiatetskiShapiro on the occasion of his sixtieth birthday, Part II, Israel Math. Conf. Proc. vol. 3, Weizmann, Jerusalem, 1990, pp. 111-125.

19. P. Lax and R. Phillips, Scattering theory for automorphic functions, Ann. of Math. Studies, 87, Princeton Univ. Press, 1976.

20. C. Moeglin and J. Waldspurger, Le spectre résiduel de $G L(n)$, Ann. Scient. Éc. Norm. Sup. 22 (1989), 605-674.

21. W. Müller, The trace class conjecture in the theory of automorphic forms II, preprint, 1996.

22. The trace class conjecture in the theory of automorphic forms, Ann. of Math. 130 (1989), 473-529.

23. __ Eigenvalue estimates for locally symmetric spaces of finite volume, in Symposium Partial Differential Equations (Holzhau, 1988), Teubner-Texte Math., 112, Teubner, Leipzig, 1989, pp. 179-196.

24. A. Reznikov, Eisenstein matrix and existence of cusp forms in rank one symmetric spaces, Geom. Funct. Anal. 3 (1993), 79-105

25. M. Osborne and G. Warner, The Selberg trace formula. V, Trans. Amer. Math. Soc. 286 (1984), 351-376.

26. _ The Selberg trace formula. II, Pacific J. Math. 106 (1983), 307-496. 
27. P. Sarnak, On cusp forms, in The Selberg trace formula and related topics, ed. by D. Hejhal et al., Contemp. Math., 53, Amer. Math. Soc., 1984, pp. 393-407.

28. A. Selberg, Harmonic analysis, in Collected papers of A. Selberg, 1, Springer-Verlag, 1989, pp. 626-647.

29. N. Wallach, On the distribution of eigenvalues of the Laplacian of a locally symmetric space, in Differential Geometry, ed. by B. Lawson, Pitman Monographs and Surveys in Pure and Appl. Math., 52, Longman Scientific \& Technical, 1991, pp.337-350.

30. G. Warner, Selberg's trace formula for nonuniform lattices: The $\mathbb{R}-$ rank 1 case, Adv. in Math. Suppl. Stud., 6, Academic Press, New York, 1979, pp. 1-142.

Department of Mathematics, University of Michigan, Ann Arbor, Mi 48109

E-mail address: lji@math.lsa.umich.edu 